\title{
A Low-Cost Versatile Broadband (DC to K-Band) Planar $50 \Omega$ Termination
}

\author{
M. O. Olbrich and E. M. Biebl
}

Technische Universität München, Fachgebiet Höchstfrequenztechnik, Arcisstr. 21, 80290 München, Germany

\begin{abstract}
Numerous standard RF circuits like directional couplers, hybrids, amplitude modulators, oscillators, etc. require a $50 \Omega$ termination. Additionally, in several measurement scenarios, ports have to be terminated. Such terminations are preferable of the same waveguide type as the circuits to avoid degradation of their performance. In general, standard fabrication steps are desired as they are costeffective. The straightforward solution is a $50 \Omega$ resistor shunted to ground by means of a via. Partial wraparound SMD resistors in a 0402 size have low parasitics making them applicable up to K-band. However, for RF, this combination suffers from the considerable inductive parasitics of the via. Different approaches to avoid the vias, with varying disadvantages exist. We analyzed the approach of broadband matching the non ideal viaresistor combination to $50 \Omega$ by means of a filter structure. The realization uses only standard design and fabrication methods. The design procedure can easily be adapted to arbitrary substrates and fabrication technologies.

The assembled $50 \Omega$ termination was measured using TRL calibration with a VNA. Therefore its characteristics are determined in the intended plane and it can be directly included into a planar $50 \Omega$ circuit. The measured terminations showed a VSWR better than 1.34 within the whole band from DC to K-band, and within $57 \%$ of the band (including the $24 \mathrm{GHz}$ ISM band) the VSWR is better than 1.22.
\end{abstract}

\section{Introduction}

The objective of the presented design was to gain a standard match for several applications that can directly be included into an actual planar design and that provides its characteristic from DC to K-band. For planar waveguides there is no standard offtheshelf solution as for coaxial cables, for instance. In fact a standard solution for planar terminations would only cover a special substrate material, and furthermore only a special thickness for the respective substrate.

For a few applications, especially at lower frequencies, a coaxial termination can be attached including a

Correspondence to: M. Olbrich

(olbrich@tum.de) coaxial-microstrip transition. Such a solution is not applicable for integrated designs. Various RF applications demand for versatile terminations that are easy to implement onto the respective waveguide plane. A typical example are amplitude modulators. They are often in need of terminating the RF power in the "off" state. Also several measurement scenarios demand for versatile terminations of residual ports.

Generally A VSWR better than 1.22 is considered to represent a perfect match, and a VSWR better than 1.43 provides the desired termination for most applications. The main focus was the $24 \mathrm{GHz}$ ISM band, but the match should be within the above mentioned limits for the whole range from DC to K-band. The design and fabrication procedure should be easily adoptable to different substrates and fabrication technologies. Finally the usual demands for low-costs as well as ease of fabrication and implementation were given.

\section{Concept}

Several concepts to implement a termination for microstrip lines have been published. Providing a virtual short by replacing a via with a matched open line (Linnér, 1986) reduces the achievable bandwidth. Attenuating the wave by placing lossy material onto the microstrip line (Thompson, 1961) is a difficulttohandle additional fabrication step. The straight forward and low-cost concept is to use standard design and fabrication steps, like vias and discrete SMD components (Lacombe, 1972). We enhance this concept with regard to the frequency range from Ku-band to K-band and in addition we determine the final circuit as well as the component characteristics in the targeted plane to get a definite specification of the termination.

Taconic TLP-5-0100 substrate was taken, the via hole was drilled manually. For the via, a $0.8 \mathrm{~mm}$ copper wire was inserted and soldered on both sides. Figure 1 shows a picture of the measured PCB with the via only and the viaresistor combination. The inductive behaviour of the measured via is apparent in the Smith chart of Fig. 2. The IMS RCX 0402PW resistor was selected because of its low parasitics due to its partial wraparound metallization. The unmatched reflection of the via-resistor combination is shown in Fig 3. Here the inductive characteristic of the via is still present and results 


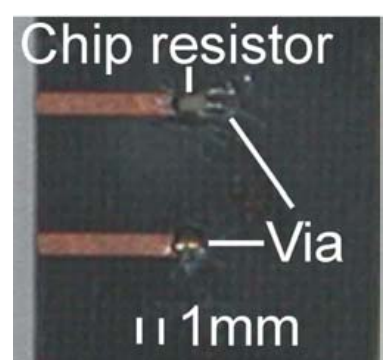

Fig. 1. Picture of the measurement PCB for the via only and the via resistor combination.

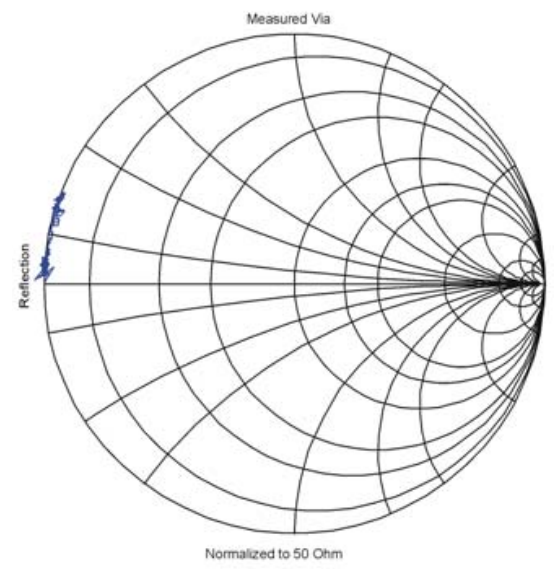

Fig. 2. Smith diagram of the measured via.

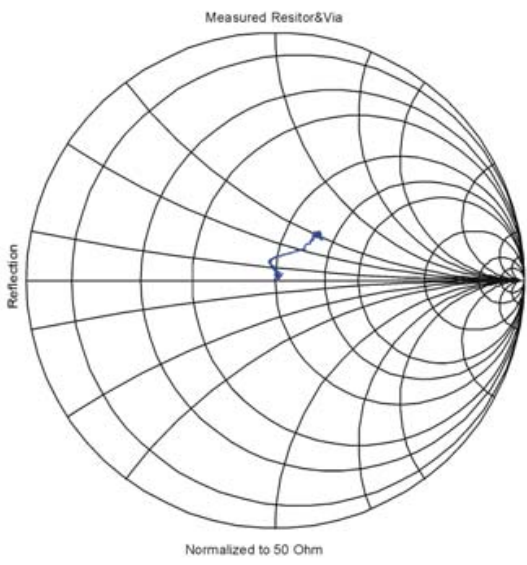

Fig. 3. Smith diagram of the measured via resistor combination.

in the poor matching of a VSWR of more than 1.43 at frequencies above $16 \mathrm{GHz}$ as shown in the magnitude plot in Fig. 4. Whereas at frequencies below $12 \mathrm{GHz}$, the matching is, as expected, already good. The inductance of the via is the critical parasitic that has to be compensated.

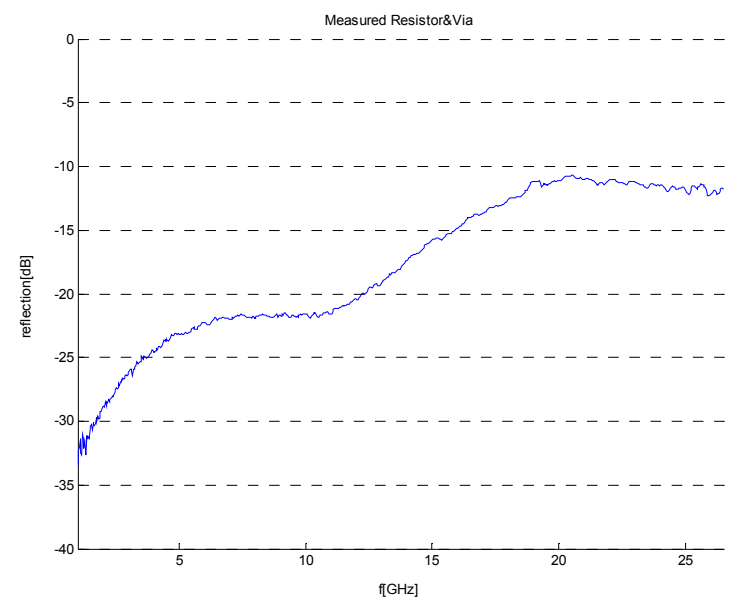

Fig. 4. Magnitude plot of the measured via resistor combination.

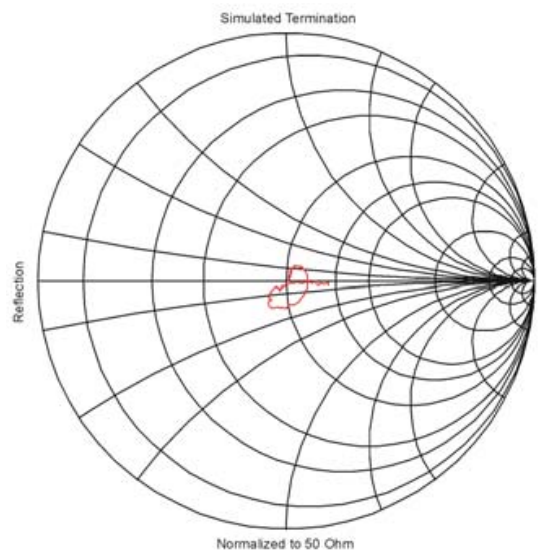

Fig. 5. Smith diagram of the simulated termination.

\section{Simulation}

Generally the parasitics of the via and the resistor limit the performance of the termination. Therefore, the reflection has to be improved by a broadband matching. The broadband matching to $50 \Omega$ was realized by means of a filter structure. Within the desired frequency range discrete components like SMD capacitors or inductors cannot be applied due to their own considerable parasitics. Since its influence is only required at frequencies above $12 \mathrm{GHz}$, a microstrip filter structure is applicable. Furthermore as the dimensions should be small anyway.

The microstrip filter structure was designed based on the method of moments. Advanced Design System (ADS) by Agilent was used as the simulation tool. The simulation is based on the above mentioned measured reflection coefficient of the via-resistor combination. Starting from an initial dimensioning, the filter was adapted by the cooptimization tool of ADS. Therewith the momentum based filter structure simulation and optimization is combined with the measured viaresistor reflection coefficient. 


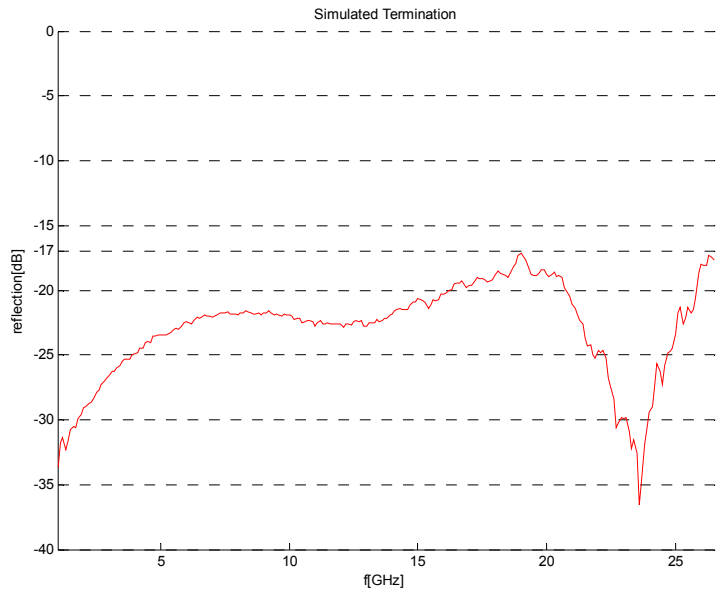

Fig. 6. Magnitude plot of the simulated termination.

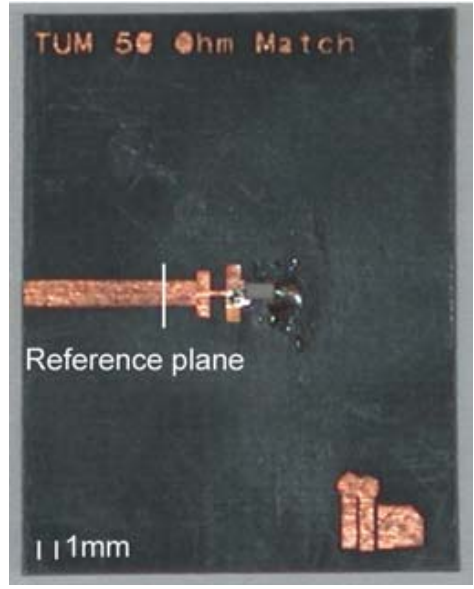

Fig. 7. Picture of the $50 \Omega$ termination.

The order of the filter was given to the simulation tool by the layout of the filter structure. Several simulations with varying filter orders showed that a $3^{r d}$ order filter provided the desired performance. The simulation results are shown in Fig. 5 in the Smith chart. The magnitude of the reflection coefficient is shown in Fig. 6. Its VSWR is better than 1.33 from DC to K-band.

\section{Measurement}

The measurement of the components as well as the measurement of the final circuit were performed with a HP 8510 VNA. The TRL calibration method was applied to achieve an onplane specification of the elements. An inhouse developed TRL calkit appropriate for the substrate and frequency range was used. To verify the calibration a passive reference DUT was applied. The onplane specification of the DUT, provided by the TRL calibration, allows the direct use of the

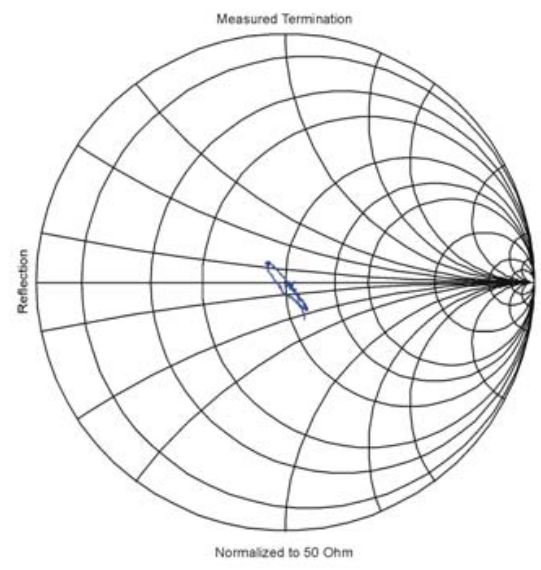

Fig. 8. Scheme of the measurement setup.

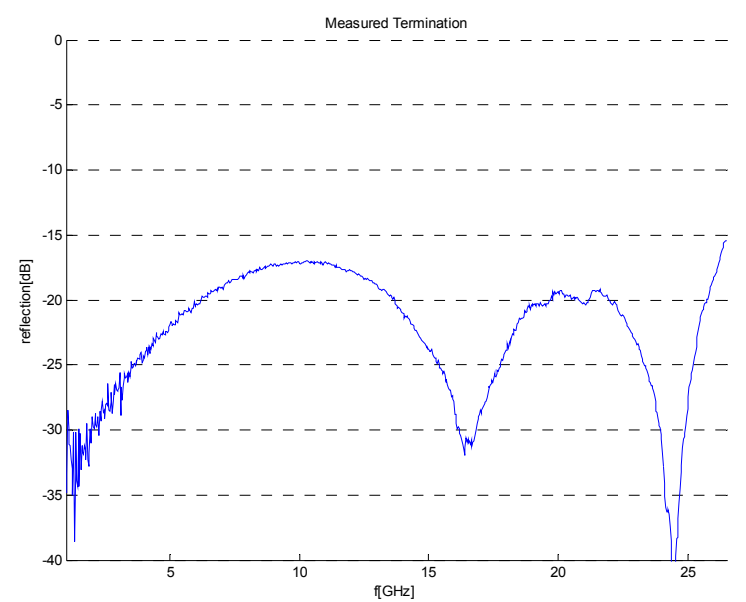

Fig. 9. ADS co optimization schematic.

measured data for the simulation and the direct connection to a $50 \Omega$ circuit of the final design.

Figure 7 shows a picture of the final circuit consisting of the via, the resistor and the microstrip filter structure. The reference plane of the measurement is set right behind the filter. At this point, the $50 \Omega$ termination can be connected to a $50 \Omega$ microstrip line of an arbitrary circuit.

The measurement showed good correspondence to the simulation results. The best matching is located in the targeted $24 \mathrm{GHz}$ ISM band. The measured data is plotted in Fig. 8 in a Smith chart. Compared to Fig. 3 the inductance of the via is compensated. The magnitude diagram in Fig. 9 shows degradation of at most $5 \mathrm{~dB}$ at frequencies below $12 \mathrm{GHz}$ compared to Figs. 4 and 6 . The second resonance is about $10 \mathrm{~dB}$ more distinctive as compared to the simulation. However, the performance is still within the desired parameters. 


\section{Conclusion}

The presented $50 \Omega$ termination has the advantage of applicability down to DC and a very broadband performance up to K-band compared to other concepts. It is based on standard design and fabrication steps that provide a low-cost and versatile implementation in production determined designs or designs for measurement purposes. The design procedure can easily be adapted to arbitrary substrates and fabrication technologies.

The realized termination shows a VSWR better than 1.34 from DC to K-band and is better than 1.22 within $57 \%$ of the range, including the $24 \mathrm{GHz}$ ISM band. Correspondence of the measurement and simulation results verifies the design process. The characteristics were determined in the intended microstrip plane and therefore the layout can be directly attached to a planar $50 \Omega$ circuit.

\section{References}

Lacombe, D.: A Multioctave Microstrip $50 \Omega$ Termination, 1972 IEEE Trans. Microwave Theory and Tech., vol. MTT-20. no. 4, 290-291, April 1972.

Linnér, P., Lundén H.: Theorie and Design of Broad-Band Nongrounded Matched Loads for Planar Circuits, 1986 IEEE Trans. Microwave Theory and Tech., vol. MTT-34. no. 8, 892-896, August 1986.

Thompson, G.H.B.: An Easy Method of Matching Microstrip Loads and Attenuators, 1961 IEEE Trans. Microwave Theory and Tech., vol. MTT-9. no. 3, 263-263, May 1961. 